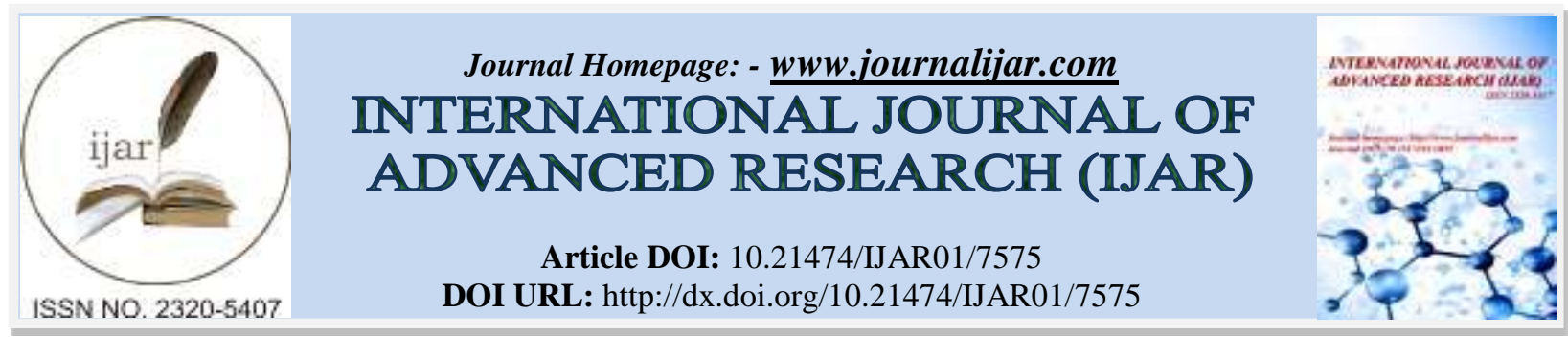

RESEARCH ARTICLE

\title{
SYNDROME DE WELLENS: DEFI DU PRATICIEN.
}

\section{A. Zbitou ${ }^{1}$, A. Chetoui ${ }^{1}$, Y. Ikejder ${ }^{2}$ and A. Khatouri ${ }^{1}$.}

1. Service de cardiologie et maladies vasculaires - Hôpital militaire Avicenne de Marrakech.

2. Service de cardiologie et maladies vasculaires - CHU Mohammed VI - Marrakech.

\section{Manuscript Info}

Manuscript History

Received: 16 June 2018

Final Accepted: 18 July 2018

Published: August 2018

\begin{abstract}
Wellens' Syndrome is a pattern of electrocardiographic T-wave changes associated with critical, proximal left anterior descending (LAD) artery stenosis associated with a high risk of myocardial infarction and sudden death. We report a case of Wellens's syndrome in a 36-year-old patient presenting with intermittent anginal chest pain. The electrocardiogram (ECG) shows a biphasic T-wave at anterior leads and Emergency angiography revealed critical LAD occlusion. It is important for the emergency physicians, to recognize the typical ECG findings of Wellens' Syndrome, because these characteristic ECG findings are considered as a marker for critical LAD occlusion.
\end{abstract}

Copy Right, IJAR, 2018, All rights reserved.

\section{Introduction:-}

Le syndrome de Wellens ou (syndrome de l'IVA) a été publié pour la première fois en 1982 par Wellens et ses collègues qui ont décrit les manifestations électrographiques chez les patients admis pour un syndrome coronarien instable caractérisé par des modifications de l'onde $\mathrm{T}$ dans les dérivations précordiales en rapport avec une sténose de l'interventriculaire proximale (1). Les critères diagnostiques de ce syndrome sont: Histoire de douleur thoracique, la non élévation des marqueurs enzymatiques ou élévation par rapport à la valeur de référence en deux temps avec segment ST isoélectrique ou sous décalé mois de $1 \mathrm{~mm}$ et absence d'une onde Q de nécrose sans rabotage de l'onde $\mathrm{R}$ avec des ondes $\mathrm{T}$ symétriques inversées ou rarement des ondes $\mathrm{T}$ diphasiques dans les dérivations antérieures (2). Le diagnostic précoce de ces patients est important car il est associé à un risque élevé d'infarctus antérieur étendu et de mort subite en l'absence de prise en charge précoce. Nous rapportons le cas d'un patient de 36 ans qui se présente pour des douleurs thoraciques intermittentes et chez qui nous avons fait le diagnostic du syndrome de Wellens.

\section{Observation:-}

Patient de 36 ans, sans facteurs de risques cardio-vasculaires, hormis un tabagisme occasionnel, sans antécédents pathologiques particuliers qui présente des douleurs thoraciques atypiques de façon intermittente depuis 7 mois. L'évolution a été marquée par l'aggravation de la douleur devenant de plus en plus importante pour des efforts de plus en plus minimes sans autres signes associés notamment pas de dyspnée ni palpitations ou syncope. A son admission le patient était asymptomatique et à l'examen physique on trouve un patient conscient stable sur le plan hémodynamique et respiratoire. L'électrocardiogramme d'admission montre une onde $\mathrm{T}$ diphasique avec inversion de la partie terminale en V2 (Figure 1). Les troponines ultrasensibles initiales sont à $30 \mathrm{ng} / \mathrm{ml}$. Le diagnostic du syndrome coronarien sans sus-décalage persistant du segment ST (NSTEMI) est retenu. Le patient a reçu la dose de charge de Clopidogrel, d'aspirine et HBPM puis adressé à la salle de cathétérisme pour coronarographie qui a

Address: - Service de cardiologie et maladies vasculaires - Hôpital militaire Avicenne de Marrakech. 
objectivé une subocclusion de l'artère interventriculaire antérieure proximale traitée par angioplastie avec mise en place d'un stent actif de 24 3,6 mm avec un bon flux TIMI (Figure 2). Un ECG de contrôle a été réalisé qui était sans anomalies (Figure 3). Une échocardiographie transthoracique a été réalisée qui a objectivé un aspect de cardiopathie hypokinétique avec dysfonction sévère du ventricule (VG) gauche avec un FEVG à $30 \%$ et un thrombus apical du VG. Le patient est déclaré sortant avec un suivi médical ordinaire.

\section{Discussion:-}

En 1979, Gerson et ses collègues décrivent la survenue, à l'effort, d'une onde T inversée dans les dérivations précordiales chez des patients présentant une sténose angiographiquement significative de l'artère interventriculaire antérieure (IVA) proximale (1). Dans cette étude, 33 des 36 patients (92\%) présentant cette anomalie électrocardiographique avaient une sténose de plus de 75\% de l'IVA proximale (3). En 1980, Gerson et Mc Henry ont étudié les résultats de l'ECG de 760 patients ayant subi une angiographie et décrivent à nouveau cette anomalie électrocardiographique, mais cette fois au repos, chez des patients qui dans $89 \%$ des cas, présentaient une sténose significative de l'IVA proximale (4). En 1982 et plusieurs fois de suite, Wellens et ses collègues ont rapporté des anomalies électrocardiographiques similaires et Tilkian était le premier à utiliser le terme de «syndrome de Wellens ", défini comme étant un ensemble de signes électrocardiographiques enregistrés au repos en l'absence de douleur angineuse chez un patient en angor instable (5).

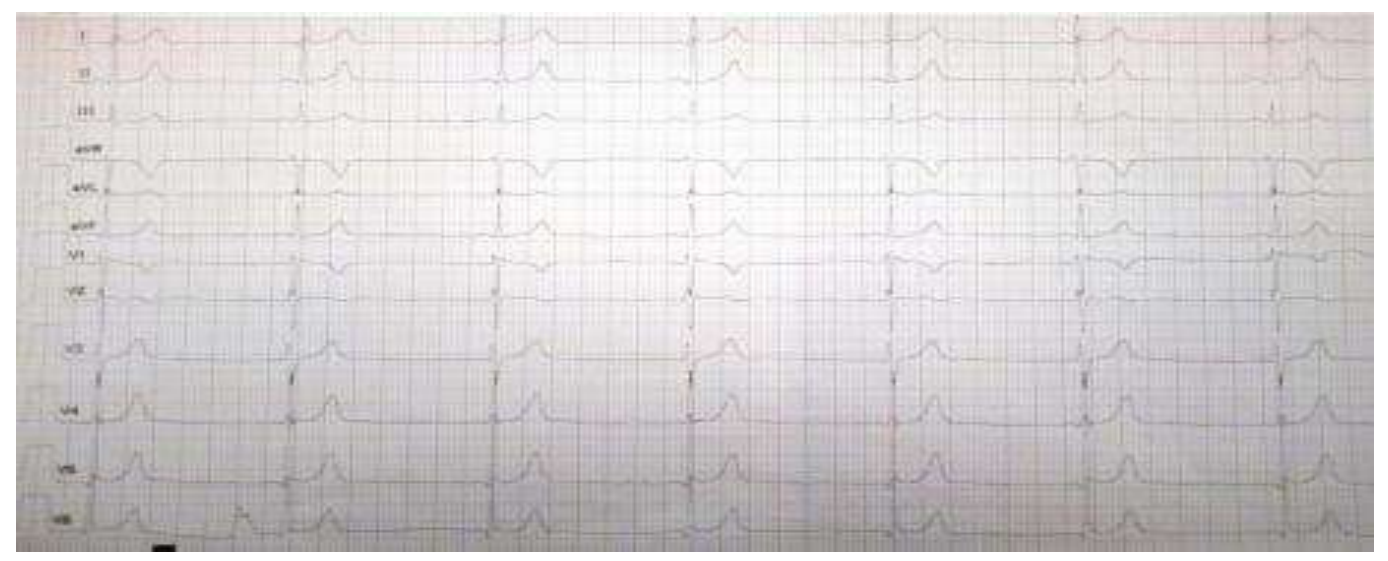

Figure 1: Onde T diphasique en V2 avec inversion de la partie terminale
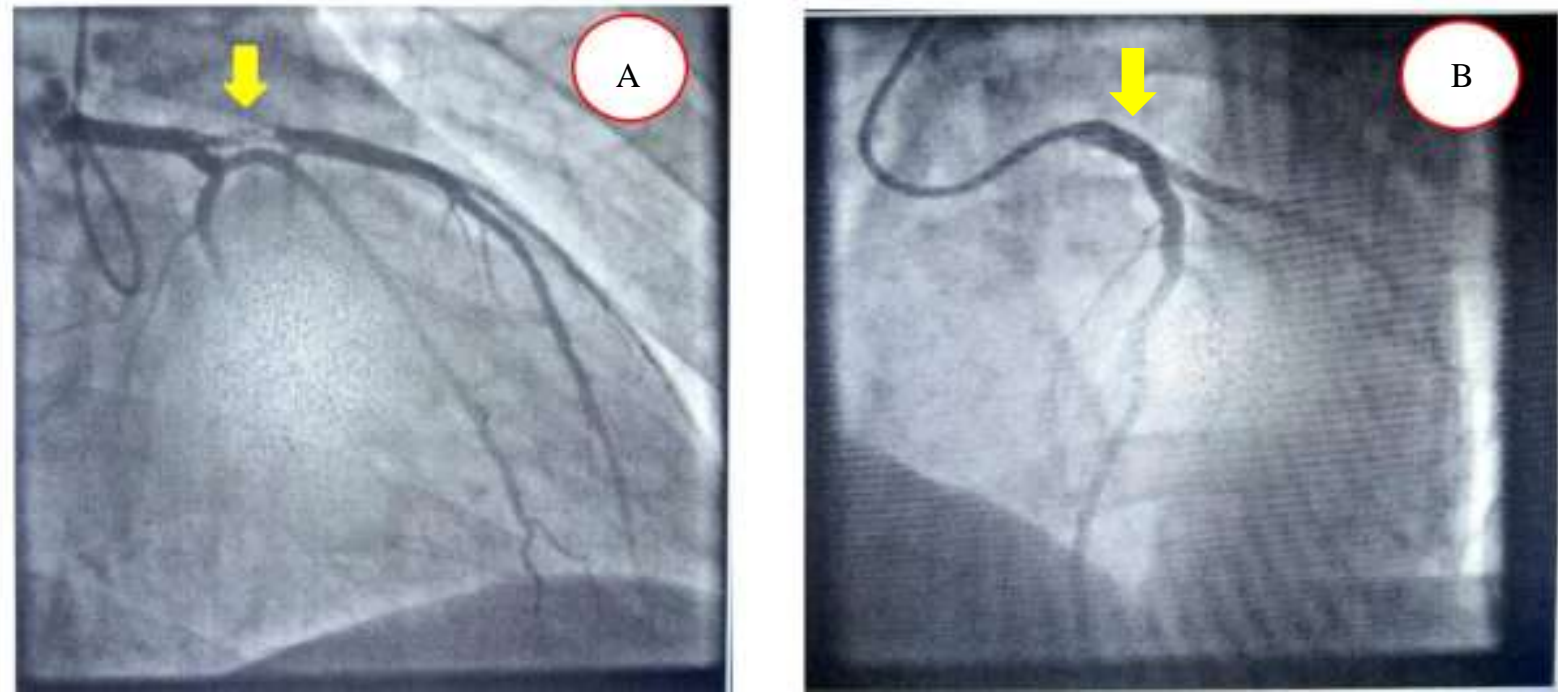

Figure 2: Coronarographie montre une sténose de $95 \%$ de l'IVA proximale (A) et le statut après angioplastie avec un bon flux (B) 


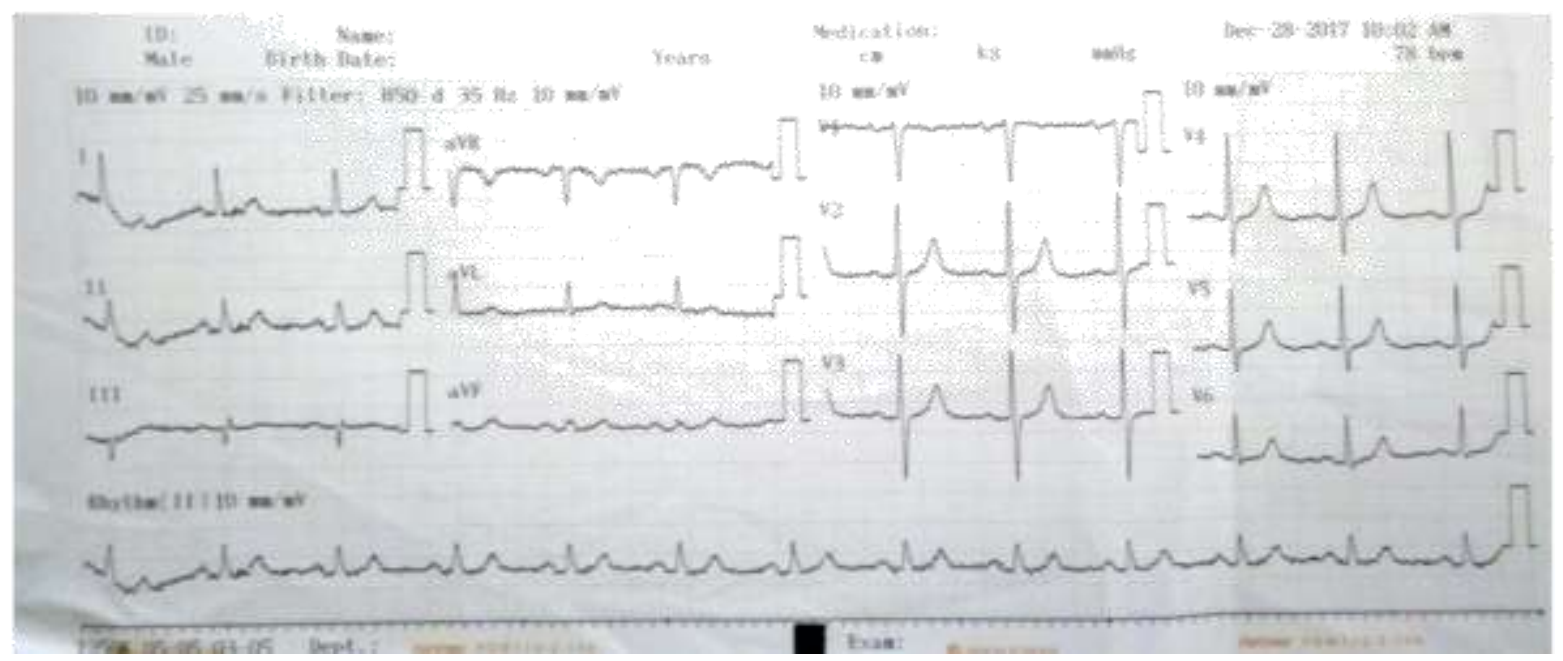

Figure 3: ECG de contrôle après angioplastie.

Dans la première étude de Wellens (5), 26 des 145 patients (18\%) admis pour angor instable présentaient cet aspect électrocardiographique, tandis que dans la $2^{\text {ème }}$ étude prospective, 180 parmi 1260 patients hospitalisés (14\%) présentaient les modifications électrocardiographiques caractéristiques. En outre tous ces patients avaient une lésion significative de l'IVA proximale. Dans la première étude 12 parmi $16(75 \%)$ des patients avec les signes électrocardiographiques caractéristiques non revascularisés avaient développé un infarctus de myocarde antérieur

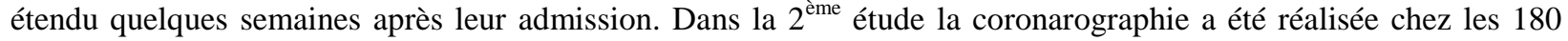
patients et qui a objectivé une sténose de l'IVA proximale et qui varie entre $50 \%$ jusqu'à une obstruction complète. La détection précoce des modifications électrocardiographiques est très importante du fait du tableau clinique atypique du syndrome de Wellens. Ces modifications électrocardiographiques sont rencontrées surtout si le patient est asymptomatique. Lorsque le patient devient symptomatique les anomalies de l'onde $\mathrm{T}$ et du segment ST se normalisent habituellement ou on peut avoir un sus décalage du segment ST. Les marqueurs enzymatiques cardiaques sont le plus souvent normaux ou légèrement élevés. Dans l'étude prospective de Wellens seulement 21 parmi 180 patients (12\%) avec les signes électrocardiographiques caractéristiques avaient une élévation des enzymes cardiaques. Celles-ci étaient toujours moins de deux fois la limite supérieure. Par conséquence l'ECG constitue le seul élément qui permet d'indiquer une exploration coronarographique en urgence. Les résultats électrocardiographiques comprennent des anomalies significatives de l'onde $\mathrm{T}$ avec des anomalies occasionnelles du segment ST. Le segment ST est le plus souvent isoélectrique ou sous décalé de moins de $1 \mathrm{~mm}$ sans rabotage de l'onde R. Wellens et ses collaborateurs (1) ont décrit alors 2 aspects ECG comme étant des facteurs prédictifs de l'existence d'une sténose critique sur l'IVA proximale (Figure 4). Ces anomalies de l'onde T peuvent également concerner V1 dans $2 / 3$ des cas et V4 dans 3/4 des cas et rarement V5 et V6. L'inversion de l'onde T est la constatation la plus fréquente à l'ECG chez les patients qui sont admis pour suspicion d'un syndrome coronarien aigu dans le service des urgences $(30 \%)$ et généralement interprété comme syndrome coronarien sans sus décalage du segment ST. Les ondes T négatives en rapport avec l'ischémie myocardique sont symétriques et profondes. Les autres pathologies qui conduisent à des ondes $\mathrm{T}$ négatives sont: les syndromes coronariens aigues (avec ou sans élévation de ST), la myocardite, l'embolie pulmonaire, l'hypertrophie ventriculaire gauche, le syndrome de WolfParkinson-White, l'onde juvénile $\mathrm{T}$ et l'effet digitalique dangereux en présence d'une lésion instable sur l'IVA proximale. 


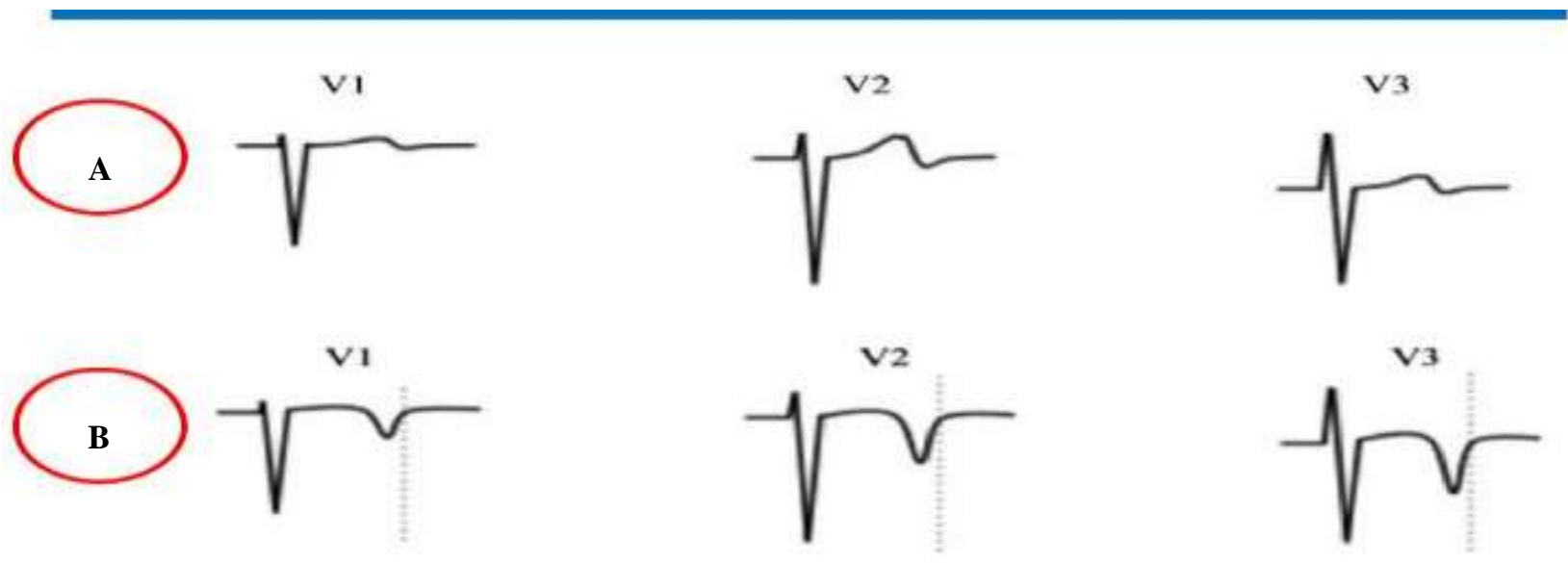

Figure 4: (A) 24\% : ondes T bi-phasiques avec inversion terminale dans les dérivations précordiales V2 et V3. (B) $76 \%$ : ondes $\mathrm{T}$ profondément inversées $(>0,2 \mathrm{mV})$ en $\mathrm{V} 2$ et $\mathrm{V} 3$, plutôt fines et symétriques. (6)

L'onde $\mathrm{T}$ diphasique est une onde qui a deux composantes commençant par une onde positive. Elle est plus spécifique à l'infarctus de myocarde que le reste des pathologies conduisant à des ondes T négatives et devrait être alarmant pour les urgentistes. Le syndrome de Wellens se réfère aux deux types de formes d'ondes $\mathrm{T}$ qui sont observées à la période indolore.

\section{Conclusion:-}

Les patients avec angor instable présentant les modifications électrocardiographiques caractéristiques du syndrome de Wellens ont un risque important de développer un infarctus du myocarde à localisation antérieure même sous traitement médical. Le cas présenté illustre la difficulté diagnostique de ce syndrome qui est largement méconnue par les praticiens. La difficulté diagnostique réside dans le polymorphisme clinique et électrocardiographique. Il faut éviter chez ces patients les évaluations ischémiques fonctionnelles qui peuvent se révéler dangereuses en présence d'une lésion instable sur l'IVA proximale (5).

\section{Références:-}

1. De Zwaan C, Bar FW, Wellens HJ: Characteristicelectrocardiographic pattern indicating acritical stenosis high in left anterior descending coronary artery in patients admitted becauseof impending myocardial infarction. Am Heart J 1982; 103: 730-736.

2. Tandy TK, Bottomy DP, Lewis JG: Wellens' syndrome. Ann Emerg Med 1999; 33: 347-351.

3. Gerson MC, Phillips JF, Morris SN, MCHenry PL. - Exercise-induced U-wave inversion as a marker of stenosis of the left anterior descending coronary artery. Circulation, 1979, 60, 1014-1020.

4. Gerson MC, McHenry PL.- Resting $\mathrm{U}$ wave inversion as a maker of stenosis of the left anterior descending coronary artery. Am J Med, 1980, 69, 545-550.

5. Une entité électrocardiographique peu connue, mais pourtant importante aux urgences... le syndrome de wellens B. Javillier,

6. C. Jacquet, V. Legrand Rev Med Liège 2012; 67: 10: 527-530 